Revista Mexicana de Economía y Finanzas, Vol. 12, No. 3, (2017), pp. 67-87

\title{
Los beneficios de la inversión socialmente responsable en el desempeño de fondos de pensiones mexicanos
}

Dr. Oscar Valdemar De la Torre Torres, M.I.S.C. ${ }^{1}$

Universidad Michoacana de San Nicolás de Hidalgo

Luis Guadalupe Macías Trejo

Universidad Michoacana de San Nicolás de Hidalgo

(Primera recepción: 31 de Mayo 2016, Segunda recepción: 21 de Septiembre 2016, Aceptado: 31 de Enero 2017.)

\section{Resumen:}

En el presente artículo se simula el impacto que tiene,en la política de inversión de las SIEFOREs,el invertir su componente de renta variable nacional en acciones mexicanas socialmente responsables, mismas que formen parte del índice de precios y cotizaciones sustentable (IPCS). Para lograr esto, se empleó la política de inversión autorizada por la CONSAR y se recalculó el IPCS a enero de 2004 con el método de capitalización. Con este índice como insumo, se simuló el comportamiento de tres SIEFOREs cuya única diferencia es emplear el IPCS, el IPCcomp y el IPC en un portafolio de mínima varianza y otro que maximiza el índice de Sharpe. Los resultados observan que, al invertir su componente de acciones en el IPCS,las SIEFOREs no pierden eficiencia media-varianza, respecto al desempeño logrado ya seacon el IPC o elIPCcomp.De manera complementaria se aprecia que si una SIEFORE utilizara el IPCS en un portafolio que maximice el índice de Sharpe durante periodos de alta volatilidad, el desempeño del mismoes mayor que el observado al emplear los otros dos índices.

Clasificación JEL: C580, G11, G170, G23.

Palabras clave: ModelosMarkovianos de cambio de régimen, Diversificación, Selección de portafolios, Simulación y pronósticofinanciero, Inversiónsocialmenteresponsable.

\section{The benefits of Socially Responsible Investment in the performance of Mexican pension funds.}

\begin{abstract}
:
In the present paper we simulate the impact of investing in socially responsible stocks (members of the IPC sustainable index or IPCS) in Mexican pension funds or SIEFOREs. In order to do this, we used the authorized investment policy by CONSAR and we recalculated the IPCS index from January 2004 by using the market cap method. With this recalculated index we simulated the performance of three SIEFOREs that invest their Mexican equity proceedings either in the IPCS, the IPCcomp or the IPC index. Our results show a sound mean-variance efficiency if the SIEFOREs invest in the IPCS index only. We
\end{abstract}

*Facultad de Contaduría y Ciencias Administrativas, Universidad Michoacana de San Nicolás de Hidalgo Ave. Francisco J. Mújica S/N Edificio AII Col. Felicitas del Río Morelia Michoacán C.P. 58030. Correo electro'nico:oscar.delatorre.torres@gmail.com

Dedicadoa mi fallecido papá: Oscar Gerardo De la Torre Martínez.

Dr. Oscar V. De la Torre Torres 
Nueva Época REMEF (TheMexicanJournal of Economics and Finance)

also found that if the SIEFOREs invest in the IPCS in a Max Sharpe portfolio, they would have a better performance in high volatility periods than investing on the other two indexes.

JEL Classification: C580, G11, G170, G23

Key words: Markov-Switching models, Diversification, Portfolio choice, Financial

forecasting and simulation, Socially responsible investment.

\section{Introducción.}

La inversión socialmente responsable es una práctica que ha tenido un auge notorio en los últimos años. Sin embargo, dista de ser una práctica profesional reciente en la historia del ser humano. Un primer ejemplo lo tenemos en la antigüedadcon la propia Torá. En la misma se establecen los tipos de actividades que era lícito realizar y, por consecuencia, en donde se podría invertir para tener una fuente de sustento. Otro ejemplo se observa con la Ley Sharia del Islam en donde se establece los productos que son "halal" o permitidos y los "harom" o prohibidos como es el invertir (y consumir) bebidas alcohólicas derivadas del trigo o la uva, así como el consumo (producción) de carne roja.

De manera formal, la inversión socialmente responsable (también denominada sustentable para fines de exposición en el presente) tiene su enfoque de estrategia de inversión y activismo con las prácticas de luteranos y metodistas, quienes buscaban hacer buen empleo del dinero, eran propensos a practicarla frugalidad como modo de vida y evitaban invertir su dinero en empresas consideradas pecaminosas según sus prácticas, costumbres y creencias(Renneboog, Ter Horts, \& Zhang, 2011). Caso de esto son las empresas productoras de armas, alcohol o tabaco. En la década de 1970, con la creación del "Paxfund" esta práctica tomó mayor fuerza y salió de la esfera religiosa, debido a que muchos inversionistas decidieron hacer activismo contra empresas involucradas en la producción de armamento e insumos en la guerra de Vietnam.

Actualmente la responsabilidad social se comprende como un concepto más amplio que rebasa la esfera religiosa, moral o ambiental y aprecia la misma como una combinación multidimensional de elementos, mismos que son resumidos en tres pilares definidos (ambiental, social y económico) gracias a la definición del milenio de la Organización de las Naciones Unidas(United Nations, 2000) y la resolución 60/1 de dicho organismo(United Nations, 2005). Partiendo de esto, el conceptode inversión socialmente responsable evolucionó de lo definido en el documento milenio previamente citado a lo que hoy en día se conoce como responsabilidad social por ESG (Enviromental, Social and Government). Se le llama así por que sus pilares son ahora el impacto ambiental y social de una empresa, así como sus estándares de gobierno corporativo.

En este punto de la introducción, es de necesidad observar que no se discutirá, en el presente artículo, lo que se comprende como "responsabilidad social corporativa" en comparación con la inversión socialmente responsable. Tampoco se discurrirá en definir cuáles son o deben ser las dimensiones (o pilares) de la inversión socialmente responsable o afirmar si el término adecuado es "sustentable" o "socialmente responsable". Esto se hace así siguiendo a Eccles y Viviers(2011) quien observan que, en términos de inversión y no en términos de prácticas corporativas, existe confusión y exactitud en el concepto de 
inversión socialmente responsable, sustentable o alguno de naturaleza similar. Lo anterior al hacer ellos una revisión de artículos en la materia y observar que el término de inversión socialmente responsable es un concepto que se origina de manera "evolutiva". Otra razón que nos motiva a hacer de lado el debate, radica en el hecho de que, como Berry y Junkus(2013) señalan, existe un amplio y heterogéneo catálogo de metodologías de evaluación de la responsabilidad social en acciones y bonos de empresas que cotizan en mercados financieros. Dado esto, el hablar de inversión socialmente responsable siguiendo el documento milenio o el ESG resulta en un ejercicio más propio de las metodologías de evaluación y de la práctica profesional; y no uno propio de situaciones filosóficas del término. Dado esto, es de necesidad resaltar que se hablará de inversión socialmente responsable, partiendo de las dimensiones contempladas en la metodología del IPC sustentable (que se sugiere sigue las dimensiones propias de la ESG en su metodología de cálculo). Esto sin discurrir qué método de valuación es el más adecuado.

Retomando el tema de interés y como resultado de este procesoevolutivo descrito, múltiples índices accionarios se han desarrollado y calculado para medir el desempeño de invertir en éste tipo de empresas (socialmente responsables). Para el caso mexicano se tiene, a partir de noviembre del año 2008, la existencia del IPC sustentable, el cual busca medir el desempeño de las acciones de emisoras que cotizan en el índice IPC composite (IPCcomp), mismas que tienen la calificación de ser sustentable y cuya membresía al índice está limitada a 35 acciones. Estas, para ser incluidas,deben cumplir con la máxima calificación de sustentabilidad, la cual es evaluada, de manera conjunta, por la Universidad Anáhuac del sur (a través de su centro de excelencia en gobierno corporativo) y la empresa Ecovalores, filial de la empresa valuadora EIRIS (EmpowermentResponsibleInvestment).

Con estos antecedentes, los términos inversión sustentable o socialmente responsable se utilizarán de forma indistinta en el presente trabajo. Dado esto, puede comenzar a verse a la inversión socialmente responsable o ISR como una nueva forma o estilo de inversión dentro de la administración de portafolios. Es decir, algo similar a las carteras con estrategias de valor ${ }^{2}$ o de crecimiento $^{3}$,propias del paso de la asignación de activos.Dado lo anterior, nos resulta de interés estudiar ya no solo el desempeño de la inversión sustentable en la Bolsa Mexicana de Valores (BMV), como se hacen en estudios previos como los de Valencia (2015), De la Torre, Galeana y Aguilasocho(2015) y De la Torre y Martínez (2015); sino su correlación con otros tipos de activos en el desempeño de una política de inversión de interés pública, como es la propia de los fondos de pensiones de contribución definida dados por la SIEFOREs. Esto lleva a plantearnos, como objetivo de investigación a resolver, la medición del impacto que tendría en el desempeño de las SIEFOREs y su eficiencia media-varianza si se emplea la inversión en acciones mexicanas socialmente responsables en su política de inversión. Como resultado de esto y de la revisión de bibliografía próxima a describir, surge la necesidad plantear la hipótesis del presente trabajo: "La inversión socialmente responsable lleva a un mejor desempeño y a una mejor relación riesgo-rendimiento en fondos de pensiones con políticas de inversión afines a la de las SIEFOREs en México".

Ante esto, el artículo comienza con una breve y no exhaustiva revisión de la bibliografía más relevante en materia de inversión socialmente responsable, así como de los

${ }^{2}$ Generación de valor a través de la recepción de dividendos y flujos.

${ }^{3}$ Inversión en acciones cuyo dividendo y valor de capitalización se espera se incremente trimestre a trimestre 
Nueva Época REMEF (TheMexicanJournal of Economics and Finance)

antecedentes que fundamentan el presente. De manera consecuente, se medirá el impacto de la inversión sustentable en la política de inversión de las SIEFOREs para, en el apartado de conclusiones, establecer si se demostró la hipótesis de trabajo, así como las guías para futuros trabajos relacionados al presente.

\section{Revisión de bibliografía.}

Desde una perspectiva internacional e histórica de los trabajos sobre inversión socialmente responsable podemos comenzar con el de Statman(2000) quien demuestra que el rendimiento del Domini Social Index es superior al del S\&P500 en el periodo 1990-1998. Sin embargo, al deflactar por riesgolos rendimientos generados (medir la relación riesgorendimiento), observa que no existe una diferencia estadísticamente significativa entre ambos. Otros de los trabajos pioneros fue el desarrollado porMoskowitz, (1972), quien encontró que se obtienen rendimientos extra en los fondos de inversión socialmente responsable, en comparación con los fondos de inversiónque aplican la denominada "inversión convencional" que consiste en invertir en acciones socialmente responsables y acciones que no tienen esta cualidad. Los resultados los justifica en virtud del mejor desempeño que tiene en su cotización las acciones de empresas socialmente responsables. Por su parteBoutin, Dufresne y Savaria(2004) realizan estudios sobre el rendimiento de los fondos que replican el índice sustentable canadiense S\&P-TSX sustentable contra fondos que emplean la estrategia de inversión convencional replicando el S\&P-TSX convencional. Los resultados a los que llegan son similares a los de Statman(2000). Más tarde Schröder(2004) desarrolla un estudio que mide el desempeño de 56 fondos de inversión sustentable de Estados Unidos, Alemania y Suiza y de 10 índices de responsabilidad social. Su trabajo mide el alpha de Jensen $(\alpha)$ de estos fondos e índices en relación a su contraparte o índice convencional de mercado, utilizando datos del 2000 al 2002. El objetivo del autor era poner a prueba el bajo rendimiento de la inversión sustentable contra los fondos del mercado buscando alphas negativas y significativas. Los resultados a los que llega no muestran evidencia estadística para soportar su hipótesis, observando que el desempeño de la inversión socialmente responsable y la convencional es estadísticamente similar.De manera complementaria, se observa lo revisado en Bauer, Koedijk y Otten(2005), quienes realizan un estudio para medir el rendimiento de los fondos de Alemania, US e Inglaterra especializados eninversión sustentable contra su índice convencional de mercado y el de inversión sustentable. Es decir, utilizan un CAPM multifactorial que emplea tanto el índice sustentable como el convencional como variables regresoras. Al emplear el modelo de Carhart(1997) para medir el desempeño y eficiencia media-varianza concluyeron, con datos mensuales de 1990 a 2001, que no existe diferencia estadísticamente significativa en el desempeño de la inversión socialmente responsable y la convencional. Incluso extienden sus conclusiones a observar que el índice sustentable, en la mayoría de los casos, no es un factor de influencia significativa; siendo el mercado convencional el más adecuado para éste fin.

Para complementar sus trabajos previos, Schröder(2007) emplea la denominada "prueba de expansión" de Hubberman y Kandel(1987) para demostrar la siguiente hipótesis: $\alpha=0, \beta=1$ en un modelo CAPM estándar utilizando en las variaciones porcentuales de 29 índices sustentables a nivel internacional como regresada y su contraparte o índice convencional como regresora. Con su trabajo, el autor busca responder tres preguntas: 1) ¿Tienen los índices sustentables un desempeño superior a los convencionales?, 2) ¿Tienen éstos índices el mismo nivel de exposición al riesgo que los convencionales? y 3) si no 
existe diferencia estadística ¿Puede la inversión socialmente responsable ser replicada en desempeño por la convencional (prueba de expansión)?. Los resultados llevan a los autores a concluir a que no existe superioridad de desempeño pero tampoco un desempeño inferior. De manera complementaria, el autor encuentra que, en 8 de los 29 índices, no se rechaza la prueba de expansión de Hubberman y Kandel, lo que lleva a observar que las relación riesgo-rendimiento entre la inversión sustentable y la convencional es estadísticamente igual.

Desde una perspectiva de un estudio de desempeño incorporando regímenes de volatilidad con modelos markovianos empleando el filtro de Hamilton (1989), Areal, Cortez y Silva (2013) estudian tres portafolios equiponderados $\left(w_{i}=1 / n\right)$. Uno que tiene un proceso de selección de acciones por medio de parámetros religiosos idóneos, otro con acciones que tienen una calificación alta en el pilar social y otro con acciones "viciosas" o no sustentables. Los autores, empleando el citado filtro de Hamilton (1989) en un modelo CAPM condicional y el modelo de Carhart (1987), llegan a observar que el fondo o portafolio vicioso tiene un mal desempeño en periodos de crisis, siendo mejores los otros dos casos creados con criterios religiosos y sociales, a pesar de su desempeño inferior, en materia de rendimiento pagado.

Para el caso mexicano se tienen algunos avances en el estudio del desempeño de la inversión sustentable. El primero de ellos es el trabajo citado deValencia (2015) quien realiza un análisis de desempeño por medio del modelo factorial de Fama y French (1992), así como el de Carhart (1997) para un portafolio equiponderado de las acciones socialmente responsables del IPC sustentable. Esto en un periodo de 1995 a $2011^{4}$. En sus conclusiones observa que el índice sustentable tiene una volatilidad menor y ofrece un menor rendimiento que el IPC. También encontró que las betas del factor momentum y el factor de capitalización son diferentes de cero y tienen una influencia en el desempeño de éste portafolio y que son cambiantes en el tiempo. De manera complementaria, se pueden citar los trabajos desarrollados por De la Torre, Galeana y Aguilasocho(2016)con datos de noviembre de 2008 a agosto de 2013, quienes estudian la eficiencia media-varianza de la inversión sustentable en México. Esto al comparar el índice IPC sustentable (IPCS) con el IPC compuesto (IPCcomp) tanto en la muestra de datos como fuera de muestra con simulaciones Monte Carlo a diez años vista. Esto bajo el supuesto de un movimiento geométrico browniano discretizado en el proceso estocástico inherente a los índices comparados. Los autores también hicieron un comparativo del nivel de volatilidad y rendimiento deflactado a riesgo con el índice de Sharpe y revisaron los resultados en fechas atípicas, las cuales identificaron con una distancia de Mahalanobis cuadrática. Sus conclusiones les llevaron a no encontrar diferencia estadística tanto en el rendimiento de ambos índices como en el nivel de riesgo y eficiencia media-varianza. Por último aplicaron la prueba de expansión de Hubberman y Kandel (1987) para determinar si el IPCcomp es un adecuado sustituto del IPCS y encontraron que la hipótesis $\alpha=0, \beta=1$ se sostenía. Por último, de manera complementaria a lo anterior, De la Torre y Martínez (2015)vuelven a realizar la prueba de expansión de Huberman y Kandel y comparan el desempeño de ambos índices aplicando el filtro de Hamilton (1989) para calcular el rendimiento medio y la desviación estándar de los rendimientos en dos regímenes de volatilidad. Sus resultados

\footnotetext{
${ }^{4}$ El autor recreó el IPC sustentable con dicho portafolio equiponderado desde enero de 1995.
} 
resaltan que, en ambos periodos, la volatilidad del IPCS es mayor que la del IPCcomp. Sin embargo, la rentabilidad del anterior es mayor que la del segundo en momentos de crisis o alta volatilidad.

Hasta ahora se ha hecho una revisión de literatura en materia del desempeño ya sea de fondos $\mathrm{o}$ de índices especializados en acciones socialmente responsables pero la incorporación de la misma, así como la medición del impacto que éste tipo de inversión tiene en el desempeño de inversionistas institucionales como fondos de pensiones es algo quees de interés reciente. Haciendo una revisión no exhaustiva adicional, se encontraron trabajos como el de Sethi (2005) quien hace una revisión a las prácticas de la inversión socialmente responsable por parte de fondos de pensiones y hace una reflexión sobre el enfoque sustentable que deben seguir estos inversionistas institucionales y el activismo que deben tener para exigir mayor transparencia, un enfoque de administración de la empresa basado en beneficios al largo plazo y un gobierno corporativo más transparente.De manera complementaria, se tiene el trabajo de Hongbo, Mitchell y Piggot (2006)quienes, de una manera análoga al presente, estudian el impacto de incluir la inversión socialmente responsable en el régimen de inversión de un fondo de pensiones. Sus pruebas las aplican para el caso japonés y observan que el incluir la inversión sustentable no lleva a ningún beneficio adicional pero si a un costo financiero (desempeño), observando que el riesgo de exposición a mercado se reduce en el portafolio.

Ante esta revisión no detalladade bibliografía, se observa que existen antecedentes que analiza la inversión socialmente responsable en bolsa para el caso internacional y mexicano, pero existe muy pocos casos que hablen del impacto que tendría la misma en fondos de pensiones. Ante esto, el presente busca contribuir a esta necesidad para el caso mexicano, al estudiar el beneficio que tendría para las SIEFOREs el implementar éste tipo de estilo de inversión. Adicional a ello, se hace un cálculo en retrospectiva del IPC sustentable y se analiza su desempeño no desde noviembre de 2008; sino desde enero de 2004. Con esto, se tiene un desarrollo tecnológico adicional que da luces del comportamiento de éste tipo de empresas en periodos de tiempo más largos (incluyendo dos crisis financieras internacionales como son la de 2007-2009 y 2011-2012).

Dada ésta necesidad detectada y solucionada en el presente, ahora se pasará a la revisión del desempeño que se lograría en la política de inversión de las SIEFOREs si incorporasen solo acciones de empresas socialmente responsables en el componente nacional de renta variable.

3. Fase experimental: medición del impacto de la inversión socialmente responsable en bolsa en el régimen de inversión de fondos de pensiones mexicanos.

Para poder medir el impacto que tiene el empleo de la inversión sustentable se utilizó la política de inversión de las SIEFORES autorizada por la CONSAR(2016), misma que se expone en la tabla 1. Como se puede apreciar en la misma, se presentan los límites de inversión mínimo y máximo por tipo de activo, así como los límites de exposición cambiaria en cada tipo de SIEFORE (de los tipos 1 a 4). Esto según el plan de inversión de tipo "ciclo de vida" diseñado para las SIEFOREs. De las mismas se obtuvo una política de inversión global que se emplearía (partiendo de las anteriores) si existiera un fondo cuyo régimen de inversión aplicase a todos los tipos de SIEFORE $^{5}$. Las restricciones de

\footnotetext{
${ }^{5}$ El estudio detallado del impacto de cada uno de los tipos de SIEFORE sale de la óptica del presente por que cada una de ellas tiene objetivos financieros diferentes y
} 
cardinalidad que se exponen en la columna de la derecha(columna "presente trabajo")serán las empleadas para resolver los problemas de optimización dados en (1) y (2) para, como consecuencia, medir el impacto de la inversión socialmente responsable sobre la política de inversión aplicable a todas las SIEFORES como régimen de inversión general. Esto deja entonces, como una futura línea de trabajos por realizar el impacto que tiene la misma sobre cada tipo de SIEFORE.

Partiendo de esta política de inversión, se puede observar que se extrajeron primero datos históricos de todos los índices citados en la tabla 1con fecha de inicio del 5 de enero del 2004. Esto es así por que la fecha de inserción del IPCcomp es a partir de éste día por lo que obtener valores de los índices desde esa fecha es factible salvo para el caso del IPC sustentable (IPCS). En éste caso lo que se hizo fue recalcular el mismo índice desde el 5 de enero del 2004 empleando como miembros (y de manera fija) las acciones que conformaban al IPCS el 28 de noviembre de 2008 que fue la fecha inicial del mismo. Con los valores de las mismas, se recalculó (ya incluyendo los miembros que año con año se agregaron por parte de la BMV) su desempeño hasta la fecha final de los datos de los otros índices que es 31 de diciembre de 2014.

Cabe señalar que los datos de los índices se extrajeron de Economatica, Bloomberg y de Yield book. Para medir el impacto de la inversión socialmente responsable, en este primer estudio trabajaremos con la medición para la inversión en acciones mexicanas. Por lo que, como se señala en la tabla 1, lo único que cambiará entre portafolios simulados será el índice de acciones mexicanas empleado que va desde un índice de mercado con los tres niveles de capitalización (small, mid y large cap) como es el IPCcomp, hasta uno de alta capitalización como el IPC y nuestra referencia socialmente responsable (IPCS). Para medir el impacto en el desempeño de cada portafolio se calcularon índices base 100 el 16 de febrero de 2004 de cada una de las tres carteras pero, para determinar las ponderaciones de cada tipo de activo permitido en la política de inversión de la tabla 1, se realizó el cálculo de la frontera eficiente observada en cada día $t$ de simulación con datos que van desde $t_{0}=5$ de enero de 2004 hasta el día $t$. Es decir, la matriz de covarianzas $\mathbf{C}$ y el vector de rendimientos esperados (e, determinados con medias aritméticas) se determinaron con un conjunto de información con una ventana temporal en la fecha de $t_{0}$ a $t$.

Una vez que se derivaron las fronteras eficientes de cada portafolio, empleando las restricciones de cardinalidad de la columna extrema derecha de la tabla 1 , se seleccionaron lo niveles de inversión del portafolio de mínima varianza que resuelve el problema de optimización dado en (1) y el que maximiza el índice de Sharpe (cartera tangente con la línea de asignación de capital) que se logra con (2), dada la tasa de interés libre de riesgo $r f^{6}$.

Para ambos casos se emplearon vectores de niveles mínimos y máximos ( a y b respectivamente) de inversión, dadas las citadas restricciones de cardinalidad de la tabla 1.

Sujeto a:

$$
\arg \min _{\mathbf{w}} \mathbf{w}^{\prime} \mathbf{C w}
$$

$\mathbf{w} \geq 0$

$\mathbf{a}<\mathbf{w}<\mathbf{b}$

en el presente solo se busca medir el impacto de la inversión sustentable en fondos de pensiones de manera general.

${ }^{6}$ Que es la tasa de CETES de 28 días vigente en el periodo $t$. 
Sujeto a:

$$
\arg \min _{\mathbf{w}} \frac{\mathbf{w}^{\prime} \mathbf{e}-r f}{\sqrt{\mathbf{w} / \mathbf{C w}}}
$$

$$
\begin{aligned}
& \mathbf{w}^{\prime} \mathbf{e}=E_{p}^{*} \\
& \mathbf{w} \geq 0 \\
& \mathbf{a}<\mathbf{w}<\mathbf{b}
\end{aligned}
$$

\begin{tabular}{|c|c|c|c|c|c|c|}
\hline $\begin{array}{l}\text { Restricción por tipo } \\
\text { de activo (min/max) }\end{array}$ & $\begin{array}{l}\text { SIEFORE } \\
\text { Básica } \\
\text { pensiones }\end{array}$ & $\begin{array}{l}\text { SIEFORE } \\
\text { Básica } 1\end{array}$ & $\begin{array}{l}\text { SIEFORE } \\
\text { Básica } 2\end{array}$ & $\begin{array}{l}\text { SIEFORE } \\
\text { Básica } 3\end{array}$ & $\begin{array}{l}\text { SIEFORE } \\
\text { Básica } 4\end{array}$ & $\begin{array}{l}\text { Presente } \\
\text { trabajo }\end{array}$ \\
\hline $\begin{array}{l}\text { Bonos emitidos por el } \\
\text { Gobierno Federal / } 1\end{array}$ & $(51 \% / 100 \%)$ & $(51 \% / 100 \%)$ & $(0 \% / 100 \%)$ & $(0 \% / 100 \%)$ & $(0 \% / 100 \%)$ & $(20 \% / 100 \%)$ \\
\hline $\begin{array}{l}\text { Bonos emitidos por el } \\
\text { Gobierno Federal en } \\
\text { USD / } 1\end{array}$ & $(51 \% / 100 \%)$ & $(51 \% / 100 \%)$ & $(0 \% / 100 \%)$ & $(0 \% / 100 \%)$ & $(0 \% / 100 \%)$ & $(2 \% / 100 \%)$ \\
\hline $\begin{array}{l}\text { Bonos corporativos } \\
\text { mexicanos } 1 /\end{array}$ & $(0 \% / 100 \%)$ & $(0 \% / 100 \%)$ & $(0 \% / 100 \%)$ & $(0 \% / 100 \%)$ & $(0 \% / 100 \%)$ & $(10 \% / 100 \%)$ \\
\hline Acciones mexicanas & $(0 \% / 5 \%)$ & $(0 \% / 5 \%)$ & $(0 \% / 25 \%)$ & $(0 \% / 30 \%)$ & $(0 \% / 40 \%)$ & $(2 \% / 40 \%)$ \\
\hline $\begin{array}{l}\text { Bonos corporativos y } \\
\text { gubernamentales } \\
\text { extranjeros 2/ }\end{array}$ & $(0 \% / 100 \%)$ & $(0 \% / 100 \%)$ & $(0 \% / 100 \%)$ & $(0 \% / 100 \%)$ & $(0 \% / 100 \%)$ & $(0 \% / 100 \%)$ \\
\hline $\begin{array}{l}\text { Mercados accionarios } \\
\text { extranjeros } 3 /\end{array}$ & $(0 \% / 5 \%)$ & $(0 \% / 5 \%)$ & $(0 \% / 25 \%)$ & $(0 \% / 30 \%)$ & $(0 \% / 40 \%)$ & $(0 \% / 40 \%)$ \\
\hline Mercancías & $0 \%$ & $0 \%$ & $(0 \% / 5 \%)$ & $(0 \% / 10 \%)$ & $(0 \% / 10 \%)$ & $(0 \% / 10 \%)$ \\
\hline $\begin{array}{c}\text { Exposición al riesgo } \\
\text { cambiario }\end{array}$ & $\begin{array}{l}\text { SIEFORE } \\
\text { Básica } \\
\text { pensiones }\end{array}$ & $\begin{array}{l}\text { SIEFORE } \\
\text { Básica } 1\end{array}$ & $\begin{array}{l}\text { SIEFORE } \\
\text { Básica } 2\end{array}$ & $\begin{array}{l}\text { SIEFORE } \\
\text { Básica } 3\end{array}$ & $\begin{array}{l}\text { SIEFORE } \\
\text { Básica } 4\end{array}$ & $\begin{array}{l}\text { Presente } \\
\text { trabajo }\end{array}$ \\
\hline $\begin{array}{l}\text { Títulos denominados en } \\
\text { moneda extranjera }\end{array}$ & $0 \%$ & $(0 \% / 20 \%)$ & $(0 \% / 20 \%)$ & $(0 \% / 20 \%)$ & $(0 \% / 20 \%)$ & $(0 \% / 20 \%)$ \\
\hline Tipo de activo & \multicolumn{2}{|c|}{ Benchmark empleado } & \multicolumn{2}{|c|}{ Proveedor } & \multicolumn{2}{|c|}{ Ticker empleado } \\
\hline $\begin{array}{l}\text { Bonos emitidos por el } \\
\text { Gobierno Federal }\end{array}$ & \multicolumn{2}{|c|}{ Valmer gubernamental } & \multicolumn{2}{|c|}{ VALMER-MSE } & \multicolumn{2}{|c|}{ MEX_Guber } \\
\hline $\begin{array}{l}\text { Bonos emitidos por el } \\
\text { Gobierno Federal en } \\
\text { USD }\end{array}$ & \multicolumn{2}{|c|}{$\begin{array}{c}\text { Cite de bonos soberanos } \\
\text { de países emergentes - } \\
\text { México }\end{array}$} & & & \multicolumn{2}{|c|}{ Citi-ESBI-MEX } \\
\hline $\begin{array}{l}\text { Bonos corporativos } \\
\text { mexicanos }\end{array}$ & \multicolumn{2}{|c|}{ Valmer corporativo } & \multicolumn{2}{|c|}{ VALMER-MSE } & \multicolumn{2}{|c|}{ MEX_CORPORATIVO } \\
\hline Acciones mexicanas & \multicolumn{2}{|c|}{$\begin{array}{l}\text { IPC / IPC composite / IPC } \\
\text { sustentable }\end{array}$} & \multicolumn{2}{|c|}{ Mexican Stock Echange } & \multicolumn{2}{|c|}{ IPyC / IPCcomp / IPCS } \\
\hline $\begin{array}{l}\text { Bonos corporativos y } \\
\text { gubernamentales } \\
\text { extranjeros }\end{array}$ & \multicolumn{2}{|c|}{$\begin{array}{l}\text { Citi de bonos mundiales } \\
\text { con grado de inversión (sin } \\
\text { MBS's) }\end{array}$} & \multicolumn{2}{|c|}{ Citigroup Inc. } & \multicolumn{2}{|c|}{ WBI } \\
\hline $\begin{array}{l}\text { Mercados accionarios } \\
\text { extranjeros }\end{array}$ & \multicolumn{2}{|c|}{ MSCI mundo gross return } & \multicolumn{2}{|c|}{ MSCI Inc. } & \multicolumn{2}{|c|}{ MSCI-Gross } \\
\hline
\end{tabular}

Tabla 1. Política de inversión autorizada por la CONSAR para las sociedades de inversión especializadas en fondos para el retiro, así como la utilizada en el presente trabajo. 
Revista Mexicana de Economía y Finanzas, Vol. 12, No. 3, (2017), pp. 67-87

Mercancías

Bloomberg-UBS

commoditty index

Bloomberg - Citigroup

UBS-com

1/ Solo activos financieros con calificación crediticia mxA o mayor.

2/ Solo activos financieros con calificación crediticia A+ o mayor.

3/ Solo a través de benchmarks autorizados por el apéndice M de CONSAR (2012).

Fuente: Elaboración partiendo de CONSAR (2016).

Tabla 2 Resumen histórico de los valores de variación porcentual, nivel de exposición al riesgo, índice de Sharpe e índice de Herfindahl-Hirschman (HH) de los portafolios simulados.

indice

Valores promedio (\% anualizado)

\begin{tabular}{lrrrc} 
empleado/portafolio Rend. MV & Rend. MS & Riesgo MV & Riesgo MS \\
\hline IPCcomp & 7.9838 & 9.4144 & 2.0687 & 4.1157 \\
IPC & 8.1334 & 10.8817 & 2.0633 & 4.2038 \\
IPCS & 8.1468 & 10.9501 & 2.0786 & 4.4856 \\
\hline
\end{tabular}

índice

Valores promedio (\% anualizado)

empleado/portafolio Sharpe MV Sharpe MS HH MV HH MS

\begin{tabular}{lcccc}
\hline IPCComp & 3.8593 & 2.2874 & 0.4835 & 0.4455 \\
IPC & 3.9419 & 2.5885 & 0.4833 & 0.4154 \\
IPCS & 3.9193 & 2.4412 & 0.4829 & 0.4205 \\
\hline \multicolumn{5}{c}{ Desviación estándar en los valores históricos } \\
\multicolumn{4}{c}{ indice } & simulados (valores anualizados en \%) \\
empleado/portafolio & Rend. MV & Rend. MS & Riesgo MV & Riesgo MS \\
\hline IPCcomp & 0.8562 & 2.3350 & 0.2748 & 3.3362 \\
IPC & 0.8602 & 3.8981 & 0.2876 & 2.9307 \\
IPCS & 0.8670 & 4.1221 & 0.2887 & 3.3732 \\
\hline
\end{tabular}

índice simulados (valores anualizados en \%)

\begin{tabular}{lcccc} 
empleado/portafolio Sharpe MV & Sharpe MS & $H H ~ M V$ & HH MS \\
\hline IPCComp & 3.1158 & 0.6999 & 0.0260 & 0.0773 \\
IPC & 2.9908 & 1.3301 & 0.0254 & 0.0770 \\
IPCS & 3.0031 & 1.2220 & 0.0269 & 0.0780 \\
\hline
\end{tabular}

índice Percentil 5\% de los valores históricos simulados

\begin{tabular}{lrrrc} 
empleado/portafolio Rend. MV & Rend. MS & Riesgo MV & Riesgo MS \\
\hline IPCcomp & 6.9483 & 7.7684 & 1.5630 & 1.6521 \\
IPC & 7.4016 & 8.0216 & 1.5497 & 1.6948 \\
IPCS & 7.4149 & 7.9500 & 1.5708 & 1.6409 \\
\hline
\end{tabular}

índice

Percentil 95\% de los valores históricos simulados

empleado/portafolio Rend. MV Rend. MS Riesgo MV Riesgo MS

\begin{tabular}{lllll}
\hline IPCcomp & $0.0358 \%$ & $0.0555 \%$ & $0.1453 \%$ & $0.7068 \%$
\end{tabular}


Nueva Época REMEF (TheMexicanJournal of Economics and Finance)

\begin{tabular}{|c|c|c|c|c|}
\hline IPC & $0.0365 \%$ & $0.0748 \%$ & $0.1454 \%$ & $0.6235 \%$ \\
\hline IPCS & $0.0366 \%$ & $0.0786 \%$ & $0.1466 \%$ & $0.6982 \%$ \\
\hline & \multicolumn{4}{|c|}{ Rendimiento acumulado en simulación (\%) } \\
\hline \multicolumn{2}{|c|}{ empleado/portafolio Rend. MV } & \multicolumn{3}{|c|}{ Rend. MS } \\
\hline IPCcomp & 130.93 & \multicolumn{3}{|c|}{180.46} \\
\hline IPC & 131.74 & \multicolumn{3}{|c|}{169.66} \\
\hline IPCS & 131.38 & \multicolumn{3}{|c|}{199.61} \\
\hline
\end{tabular}

Aquí se presentanlas medidas de resumen de los tres portafolios simulados. Cada portafolio o SIEFORE simulada se titula según el índice accionario empleado IPCcomp para la SIEFORE que empleó dicho índice y así consecutivamente, así como MV para el portafolio de mínima varianza, así como MS el máximo índice de Sharpe..

Fuente: Elaboración propia en base a las simulaciones realizadas.

Una vez que se lograron las ponderaciones $\mathbf{w}$ observadas en $t$ para cada uno de los dos portafolios simulados, se emplearon las mismas para calcular el índice valor 100 de cada uno de los tres casos cuya única diferencia es el empleo ya sea del IPCcomp, el IPC o el IPCS.

Como se puede apreciar en el primer segmento de la tabla 2, la cartera que tuvo (en promedio) la mayor variación porcentual promedio o esperada fue la que empleó acciones mexicanas sustentables (IPCS).Esto para el caso que maximiza el índice de Sharpe (MS), siendo un comportamiento muy cerrado entre ésta y el IPC convencional para la cartera que utiliza la mínima varianza (MV). De manera análoga, se puede apreciar que el nivel promedio de riesgo en el portafolio MS que emplea el IPC sustentable es el más alto, siendo los niveles de riesgo de las tres carteras MV muy similar. Sin embargo, se puede apreciar que, al calcular el índice de Sharpe observado diariamente, el empleo del IPC convencional (IPC) lleva a los valores más altos, seguido del que emplea la inversión sustentable. Por último, se puede apreciar que, tanto en las carteras MV como MS, se tienen niveles de concentración altos (superiores a 0.15), medidos estos con el índice de Herfindahl-Hirschmann (HH).

Para afinar el análisis un poco más, se presentan las tablas 3 y 4 que exponen la prueba ANOVA unidireccional de las variaciones porcentuales e índices de Sharpe de las carteras MS resumidos en la tabla 2. En las mismas se aprecia que, a pesar de que la figura $1 \mathrm{y}$ la tabla 2 sugieren un desempeño similar para los portafolios simulados, las prueba ANOVA concluyen lo contrario.

Tabla 3 Prueba ANOVA unidireccional de la variación porcentual de los portafolios simulados que maximizan el índice de Sharpe (MS).

\begin{tabular}{ccccccc}
\hline $\begin{array}{c}\text { Origen de las } \\
\text { variaciones }\end{array}$ & $\begin{array}{c}\text { Suma de } \\
\text { cuadrados }\end{array}$ & $\begin{array}{c}\text { Grados de } \\
\text { libertad }\end{array}$ & $\begin{array}{c}\text { Promedio de los } \\
\text { cuadrados }\end{array}$ & Estadístico F & Probabilidad & $\begin{array}{c}\text { Valor crítico } \\
\text { para F }\end{array}$ \\
\hline Entre grupos & $0.0007 \%$ & 2 & $0.0003 \%$ & 164.5498 & $0.0000 \%$ & 2.9968 \\
& & & & & & \\
$\begin{array}{c}\text { Dentro de los } \\
\text { grupos }\end{array}$ & $0.0165 \%$ & 8226 & $0.0000 \%$ & & & \\
$\quad$ Total & $0.0172 \%$ & 8228 & & & & \\
\hline
\end{tabular}

Fuente: Elaboración propia en base a las simulaciones realizadas. 
Tabla 4 Prueba ANOVA unidireccional del índice de Sharpe de los portafolios simulados que maximizan el índice de Sharpe (MS).

\begin{tabular}{|c|c|c|c|c|c|c|}
\hline $\begin{array}{c}\text { Origen de las } \\
\text { variaciones }\end{array}$ & $\begin{array}{c}\text { Suma de } \\
\text { cuadrados }\end{array}$ & $\begin{array}{c}\text { Grados de } \\
\text { libertad }\end{array}$ & $\begin{array}{l}\text { Promedio de } \\
\text { los cuadrados }\end{array}$ & $\begin{array}{c}\text { Estadístico } \\
F\end{array}$ & Probabilidad & $\begin{array}{c}\text { Valor crítico } \\
\text { para } F\end{array}$ \\
\hline Entre grupos & 0.4320 & 2.0000 & 0.2160 & 28.9731 & $0.0000 \%$ & 2.9968 \\
\hline $\begin{array}{c}\text { Dentro de los } \\
\text { grupos }\end{array}$ & 61.3277 & $8,226.0000$ & 0.0075 & & & \\
\hline Total & 61.7597 & $8,228.0000$ & & & & \\
\hline
\end{tabular}

Fuente: Elaboración propia en base a las simulaciones realizadas.

De manera complementaria y para dar una explicación de los resultados observados, se presenta, en la tabla 5, el resumen de los niveles de inversión en cada tipo de mercado para las carteras simuladas. Como puede apreciarse en la misma y como resultado de la política de inversión expresada en la tabla 1, se nota para los portafolios MS que los activos que presentan mayor concentración son la deuda nacional y extranjera, seguida de la inversión en acciones mexicanas. Sin embargo, para las SIEFOREs que emplearon los portafolios de mínima varianza (MV), la inversión en acciones mexicanas es sistemáticamente menor (solo 2\%), lo que justifica la relativa homogeneidad de los resultados en estos casos y deja, como foco de atención central, las carteras MS M $^{7}$

Una situación que debe tomarse en consideración del análisis realizado es el hecho de que, para los resultados previos se está empleando toda la muestra de simulaciones desde el año 2004 hasta el 2014. Ante esto, no se puede distinguir si los mismos se sostienen en periodos de crisis o no. Para poder revisar esto se empleó el filtro de Hamilton (1989) suponiendo que el comportamiento del proceso estocástico inherente a los portafolios simulados corresponde a uno de dos regímenes de volatilidad modelados a través de una cadena markoviana de primer orden. Esto es:

$$
\Delta \%_{p, t} \sim \Phi\left(E\left(\Delta \%_{p, t}\right)_{S}, \sigma^{2}\left(\Delta \%_{p, t}\right)_{S}\right)
$$

En la expresión anterior, se observa que existe un rendimiento esperado (promedio) y una varianza que corresponde a las variaciones porcentuales $\left(\Delta \%_{p, t}\right)$ del portafolio de interés correspondiente al régimen $S$ de volatilidad. Esto lleva a determinar dos medias $\left(E\left(\Delta \%_{p, t}\right)_{S}\right)$ o rendimientos esperados y dos varianzas $\left(\sigma^{2}\left(\Delta \%_{p, t}\right)_{S}\right)$. Un par de parámetros para un régimen de baja volatilidad en los mercados objeto de inversión $(\mathrm{S}=1)$ y en otro para correspondiente a los periodos de "crisis" o volatilidad alta $(S=2)$.

Tabla 5 Resumen de los niveles de inversión por tipo de activo en los portafolios simulados.

\begin{tabular}{lrrrrrrr}
\hline & \multicolumn{7}{c}{ Mínima varianza $(\mathbf{M V})$ : promedio de niveles de inversión observados en t $(\%)$} \\
\hline Portafolio & IPCcomp- & & Citi-ESBI- & MSCI- & UBS- \\
simulado & IPC-IPCS & MEX_Guber MEX_CORPORATIVO & MEX & Citi-WBI & Gross & Bcomm \\
IPCcomp & 2.0000 & 14.3917 & 63.6083 & 8.9757 & 6.9210 & 2.6702 & 1.4331 \\
IPC & 2.0000 & 14.1903 & 63.8097 & 8.9413 & 7.3085 & 2.2870 & 1.4632
\end{tabular}

${ }^{7}$ Que son consistentes con lo establecido en la teoría financiera en materia de ser el proxy teórico de la "cartera de mercado". Favor de referirse a Bodieet. al.(2014) para mayor detalle introductorio en esta afirmación. 
Nueva Época REMEF (TheMexicanJournal of Economics and Finance)

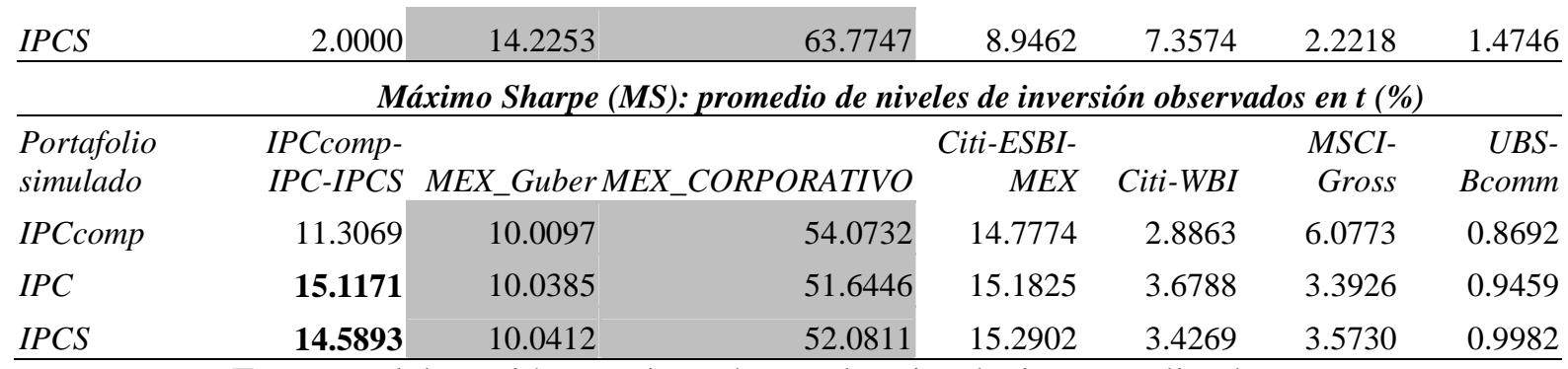

Fuente: Elaboración propia en base a las simulaciones realizadas.

Los resultados de éste análisis se exponen en la tabla $6^{8}$. En la misma se aprecia con mayor detalle la causa que genera los resultados de las tablas 3 y 4 . Como punto de partida, se señalan los valores observados para los portafolios que emplearon la cartera de mínima varianza o MV.Se aprecia que, en el periodo de baja volatilidad, emplear el IPCS lleva al mayor rendimiento esperado en las tres carteras simuladas, así como al mayor riesgo. Sin embargo, en periodos de alta volatilidad el rendimiento medio logrado sería mayor con el IPC. Esto lleva a niveles de eficiencia media-varianza (índices de Sharpe) mayores al emplear el IPC. Por lo tanto, si las SIEFORES emplearan un portafolio de mínima varianza, sería más idóneo utilizar el IPC como componente de renta variable. Sin embargo, los resultados se tornan mixtos al utilizar el portafolio que maximiza el índice se Sharpe (MS). Se puede apreciar que en tiempos de baja volatilidad resulta atractivo invertir en el IPC pero, en tiempos de alta volatilidad, es mejor hacerlo en el IPCS ya que la eficiencia mediavarianza es mayor. Esto sugiere, como línea de futuras investigaciones, el incluso estar intercambiando posiciones entre IPC e IPCS según el régimen de volatilidad vigente en $t$.

Tabla 6 Tabla de los parámetros observados al aplicar el filtro de Hamilton (1989) en los portafolios simulados.

Mínima varianza (MV): Filtro de Hamilton de variaciones porcentuales con dos regímenes de comportamiento (\% anualizado)

\begin{tabular}{|c|c|c|c|c|c|c|}
\hline $\begin{array}{l}\text { Portafolio que } \\
\text { emplea el índice }\end{array}$ & $\begin{array}{l}\text { Media reg. } \\
\text { Baja vol. }\end{array}$ & $\begin{array}{l}\text { Media reg. } \\
\text { Alta vol. }\end{array}$ & $\begin{array}{l}\text { Desv. Est. } \\
\text { reg. Baja } \\
\text { vol. }\end{array}$ & $\begin{array}{l}\text { Desv. Est. } \\
\text { reg. Alta vol. }\end{array}$ & $\begin{array}{c}\text { Sharpe Bajc } \\
\text { vol. }\end{array}$ & $\begin{array}{c}\text { Sharpe } \\
\text { Alta } \\
\text { vol. }\end{array}$ \\
\hline IPCS & 7.9938 & 6.6450 & 1.4957 & 3.7711 & 2.4447 & 0.4515 \\
\hline IPCcomp & 7.9543 & 6.7029 & 1.4590 & 3.7780 & 2.4791 & 0.4660 \\
\hline$I P C$ & 7.9918 & 6.7819 & 1.4538 & 3.6831 & 2.5138 & 0.4995 \\
\hline
\end{tabular}

Máximo Sharpe (MS): Filtro de Hamilton de variaciones porcentuales con dos regímenes de comportamiento (\% anualizado)

\begin{tabular}{|c|c|c|c|c|c|c|}
\hline $\begin{array}{l}\text { Portafolio que } \\
\text { emplea el índice }\end{array}$ & $\begin{array}{c}\text { Media reg. } \\
\text { Baja vol }\end{array}$ & $\begin{array}{l}\text { Media reg. } \\
\text { Alta vol. }\end{array}$ & $\begin{array}{l}\text { Desv. Est. } \\
\text { reg. Baja } \\
\text { vol. }\end{array}$ & $\begin{array}{l}\text { Desv. Est. } \\
\text { reg. Alta vol. }\end{array}$ & $\begin{array}{c}\text { Sharpe Bajo } \\
\text { vol. }\end{array}$ & $\begin{array}{c}\text { Sharpe } \\
\text { Alta } \\
\text { vol. }\end{array}$ \\
\hline IPCS & 9.7167 & 11.5628 & 2.2650 & 11.8644 & 2.3751 & 0.5580 \\
\hline IPCcomp & 9.0632 & 10.9386 & 1.9646 & 8.9512 & 2.4055 & 0.6699 \\
\hline
\end{tabular}

${ }^{8}$ Se presentó el valor promedio de la tasa de CETES de 28 días observado en periodos de alta y baja volatilidad. Esto para calcula el índice de Sharpe. 
Revista Mexicana de Economía y Finanzas, Vol. 12, No. 3, (2017), pp. 67-87

\begin{tabular}{ccccccc} 
IPC & $\mathbf{1 0 . 6 0 8 2}$ & 4.9974 & 2.1371 & 9.8941 & $\mathbf{2 . 9 3 4 4}$ & 0.0056 \\
\hline \multicolumn{5}{c}{ Valor de la tasa de CETES (\% anualizada) con el filtro de Hamilton. } \\
\hline $\begin{array}{c}\text { Media reg. } \\
\text { Baja vol }\end{array}$ & $\begin{array}{c}\text { Media reg. } \\
\text { Alta vol. }\end{array}$ \\
$\begin{array}{c}\text { CETES } \\
\text { promedio }\end{array}$ & & & \\
(tasa mercado) & 4.3372 & 4.9424 &
\end{tabular}

Fuente: Elaboración propia en base a las simulaciones realizadas.

Como causa de lo anterior, surge la pregunta de si el análisis empleado es realmente necesario. Es decir, si existen dos regímenes de volatilidad en las series de tiempo de los tres portafolios simulados, así como en los activos que los conforman. Para responder esto, Se realizaron dos pruebas no lineales de bondad de ajuste. La primera de ellas fue calcular la función de verosimilitud lograda en las variaciones porcentuales de los activos y portafolios simulados, suponiendo dos posibles escenarios. El primero de ellos con una función de verosimilitud gaussiana unimodal (convencional) dada por:

$$
\Delta \%_{p, t} \sim \Phi\left(E\left(\Delta \%_{p, t}\right), \sigma^{2}\left(\Delta \%_{p, t}\right)\right)
$$

El segundo escenario se modela con una función de probabilidad dada por una mixtura o combinación gaussiana bimodal:

$$
\begin{aligned}
\Delta \%_{p, t} \sim \pi\left[\Phi\left(E\left(\Delta \%_{p, t}\right)_{S=1}, \sigma^{2}\left(\Delta \%_{p, t}\right)_{S=1}\right)\right]+ \\
(1-\pi)\left[\Phi\left(E\left(\Delta \%_{p, t}\right)_{S=2}, \sigma^{2}\left(\Delta \%_{p, t}\right)_{S=2}\right)\right]
\end{aligned}
$$

En la expresión anterior se aprecia que, a diferencia del modelo markoviano con cambio de régimen dado en (3) y en (4), se presupone que la función de verosimilitud modela el comportamiento aleatorio de una población bimodal o con dos partes $(S=1,2)$, cuya proporción de los datos $(\pi)$ del primer segmento $(S=1)$ es fija. Este escenario se modela así por que las funciones de mixturas o combinaciones gaussianas son un caso especial de los modelos markovianos de cambio de régimen (Hamilton, 1994) con función de verosimilitud gaussiana ${ }^{9}$. Esto es así por que dichas funciones de mixturas calculan parámetros aplicables aplicables a todos los periodos de volatilidad; contrario al filtro de Hamilton (1989) que determina un parámetro según la probabilidad de encontrarse (en $t$ ) en el régimen de baja volatilidad o el de alta.

Tabla 7 Pruebas no lineales (coeficiente de información de Schwartz) para determinar la pertinencia del uso del filtro de Hamilton (1989) en los portafolios simulados y los índices objeto de inversión.

\begin{tabular}{cccc}
$\begin{array}{c}\text { Índice empleado } \\
\text { en el portafolio } \\
\text { simulado }\end{array}$ & $\begin{array}{c}\text { Activo/portafolio } \\
\text { simulado }\end{array}$ & $\begin{array}{c}\text { Probabilidad } \\
\text { gaussiana } \\
\text { unimodal }\end{array}$ & $\begin{array}{c}\text { Probabilidad } \\
\text { gaussiana } \\
\text { bimodal }\end{array}$ \\
\hline & Min variance IPCS & $-28,161.99$ & $-28,661.19$ \\
IPCS & Max Sharpe IPCS & $-22,683.72$ & $-24,503.23$
\end{tabular}

${ }^{9}$ Que es el caso empleado en el filtro de Hamilton en el presente trabajo. Otras funciones de verosimilitud en dicho filtro, como la t-Student o la Generalizada de valores extremos, se dejan como una recomendación para futuros trabajos de investigación. 
Nueva Época REMEF (TheMexicanJournal of Economics and Finance)

\begin{tabular}{cccc}
\hline $\begin{array}{c}\text { Índice empleado } \\
\text { en el portafolio } \\
\text { simulado }\end{array}$ & $\begin{array}{c}\text { Activo / portafolio } \\
\text { simulado }\end{array}$ & $\begin{array}{c}\text { Probabilidad } \\
\text { gaussiana } \\
\text { unimodal }\end{array}$ & $\begin{array}{c}\text { Probabilidad } \\
\text { gaussiana } \\
\text { bimodal }\end{array}$ \\
\hline \multirow{4}{*}{ IPCcomp } & Min variance IPCcomp & $-28,208.25$ & $-28,746.76$ \\
& Max Sharpe IPCcomp & $-24,122.54$ & $-25,578.93$ \\
IPC & Min variance IPC & $-28,212.85$ & $-28,733.70$ \\
& Max Sharpe IPC & $-23,403.26$ & $-24,877.73$ \\
& IPCcomp & $-15,746.65$ & $-16,676.88$ \\
& IPCS & $-15,318.92$ & $-15,910.57$ \\
& IPC & $-16,017.55$ & $-16,536.99$ \\
& MEX_Guber & $-27,388.20$ & $-28,584.58$ \\
& MEX_CORPORATIVO & $-29,414.01$ & $-30,353.69$ \\
& Citi-ESBI-MEX & $-19,417.26$ & $-20,067.68$ \\
Citi-WBI & $-18,991.34$ & $-19,540.79$ \\
& MSCI-Gross & $-18,093.40$ & $-18,750.58$ \\
& UBS-BComm & $-17,153.82$ & $-17,281.43$ \\
\hline
\end{tabular}

Fuente: Elaboración propia en base a las simulaciones realizadas.

Una vez que se lograron las funciones de verosimilitud para cada activo y portafolio en cada escenario, se calculó con ellas el criterio de información de Schwartz (por ser el más exhaustivo con la parsimonia del modelo econométrico) y se comparó el valor de las mismas. Los resultados de esta prueba de presentan en la tabla 7. En la misma se puede apreciar que suponer que la función de probabilidad es bimodal es adecuado tanto para los activos que conforman los portafolios simulados como en las propias carteras.

Dado este resultado expuesto en las tablas 4 y 7, así como lo expuesto en los niveles de inversión promedio de la tabla 5, surge la curiosidad de saber, qué mercados, de los que son objeto de inversión para las SIEFOREs, son los que más influyen en el desempeño de los portafolios simulados. Esto para determinar su influencia y potencial covarianza con los demás. Ante esto, se volvió a aplicar el filtro de Hamilton (1989) en el siguiente modelo CAPM multifactorial que ayudará a realizar un análisis de atribuciones en periodos de crisis y no crisis (para consultar el significado de cada variable regresora, favor de referirse a los códigos o tickers empleados para cada tipo de mercado en la tabla 1):

$\Delta \%_{p, t, S}=\alpha_{S}+\beta_{1, S} I P C \operatorname{comp} / I P C / I P C S+\beta_{2, S} M E X_{-} G U B E R+\beta_{3, S} M E X_{-} C O R P O R A T I V O+$ $\beta_{4, S} C I T I_{-} E S B I_{-} M E X+\beta_{5, S} C I T I_{-} W B I+\beta_{6, S} M S C I_{-} G R O S S+\beta_{7, S} U B S_{-} B C O M M+\varepsilon_{t, S}$

La razón que motiva el empleo del filtro de Hamilton en el modelo anterior surge de nuevo con los resultados de la tabla 7. Esto es así por que se entiende que la variación porcentual de los portafolios simulados es una transformación lineal de los activos que conforman dicha cartera y los niveles de inversión en cada uno $\left(w_{i}\right)$ dada por:

$$
\Delta \%_{p, t}=\sum_{i=1}^{N} w_{i} \cdot \Delta \%_{i, t}
$$

Los resultados del análisis realizado en (6) para los dos portafolios simulados se exponen en la tabla 8. En la misma se puede apreciar, para el caso de los portafolios MV, que los activos que mayor influencia son los bonos gubernamentales y corporativos mexicanos denominados en moneda nacional, así como los gubernamentales mexicanos denominados 
Revista Mexicana de Economía y Finanzas, Vol. 12, No. 3, (2017), pp. 67-87

en moneda dólares de los Estrados Unidos. Esto surge en línea con lo observado en los niveles de inversión promedio de la tabla 5. Sin embargo, en periodos de alta volatilidad, solo los bonos mexicanos denominados en dólares, las acciones internacionales (MSCI) y las mercancías o commoditties influyen significativamente en el desempeño de los portafolios simulados. 
Nueva Época REMEF (TheMexicanJournal of Economics and Finance)

Tabla 8 Tabla de los parámetros del modelo CAPM multifactorial determinado al aplicar el filtro de Hamilton (1989) con dos regímenes de volatilidad en las variaciones porcentuales de los portafolios simulados.

\begin{tabular}{|c|c|c|c|c|c|c|c|c|c|}
\hline \multicolumn{10}{|c|}{ Filtro de Hamilton con modelo de factores para portafolio de mínima varianza (régimen de baja volatilidad) } \\
\hline $\begin{array}{l}\text { Portafolio } \\
\text { simulado }\end{array}$ & Constante & $\begin{array}{r}\text { Acciones } \\
\text { Mexico }\end{array}$ & MEX_Guber & $\begin{array}{r}M E X_{-} \\
\text {CORPORATIVO }\end{array}$ & $\begin{array}{r}\text { Citi-ESBI- } \\
M E X\end{array}$ & Citi-WBI & MSCI-Gross & UBS-Bcomm & d. error. \\
\hline IPCcomp & $0 * * *$ & 0.0197 & 0.0981 & 0.6767 & 0.082 & 0.077 & 0.0249 & 0.0118 & $0.0002 \%$ \\
\hline$I P C$ & 0 & 0.0196 & 0.1005 & 0.6821 & 0.0821 & 0.0789 & 0.0225 & 0.0123 & $0.0001 \%$ \\
\hline$I P C S$ & 0 & 0.0196 & 0.0985 & 0.6822 & 0.0808 & 0.0809 & 0.0216 & 0.0126 & $0.0001 \%$ \\
\hline
\end{tabular}

Filtro de Hamilton con modelo de factores para portafolio de mínima varianza (régimen de alta volatilidad)

\begin{tabular}{|c|c|c|c|c|c|c|c|c|c|}
\hline $\begin{array}{l}\text { Portafolio } \\
\text { simulado }\end{array}$ & Constante & $\begin{array}{r}\text { Acciones } \\
\text { Mexico }\end{array}$ & MEX_Guber & $\begin{array}{r}\text { MEX } \\
\text { CORPORATIVO }\end{array}$ & $\begin{array}{r}\text { Citi-ESBI- } \\
M E X\end{array}$ & $C i t i-W B I$ & MSCI-Gross & \multicolumn{2}{|c|}{ UBS-Bcomm Std. error. } \\
\hline IPCcomp & $0 * * *$ & $0.0402 * * *$ & $-0.5225 * * *$ & $0.3229 * * *$ & $-0.1815^{* * *}$ & $0.0826 * * *$ & $0.077 * *$ & $0.0853 * *$ & $0.2250 \%$ \\
\hline$I P C$ & $0 * * *$ & $-0.0051 * * *$ & $-0.2428 * * *$ & $-1.4768^{* *}$ & 0.1696 & $-0.0537 *$ & $0.054 *$ & $-0.0231 * * *$ & $0.0613 \%$ \\
\hline IPCS & $0 * * *$ & $0.0128 * * *$ & $-0.0778 * * *$ & $0.1447 * *$ & 0.0902 & $0.0127 * * *$ & -0.0856 & 0.0543 & $0.1059 \%$ \\
\hline
\end{tabular}

Filtro de Hamilton con modelo de factores para portafolio de Máximo Sharpe (régimen de baja volatilidad)

\begin{tabular}{|c|c|c|c|c|c|c|c|c|c|}
\hline $\begin{array}{l}\text { Portafolio } \\
\text { simulado }\end{array}$ & Constante & $\begin{array}{r}\text { Acciones } \\
\text { Mexico }\end{array}$ & MEX_Guber & $\begin{array}{r}\text { MEX } \\
\text { CORPORATIVO }\end{array}$ & $\begin{array}{r}\text { Citi-ESBI- } \\
M E X\end{array}$ & Citi-WBI & MSCI-Gross & \multicolumn{2}{|c|}{ UBS-Bcomm Std. error. } \\
\hline IPCсотр & 0 & 0.0219 & 0.1594 & 0.5915 & 0.1252 & 0.0571 & 0.0181 & $0.0007 * * *$ & $0.0006 \%$ \\
\hline$I P C$ & 0.0001 & 0.0431 & 0.0825 & 0.6347 & 0.1196 & 0.063 & -0.0047 & 0.0187 & $0.0014 \%$ \\
\hline IPCS & 0.0001 & 0.0336 & 0.0826 & 0.6724 & 0.1288 & 0.0581 & $0.0022 * *$ & 0.0074 & $0.0007 \%$ \\
\hline
\end{tabular}

Filtro de Hamilton con modelo de factores para portafolio de Máximo Sharpe (régimen de alta volatilidad)

\begin{tabular}{|c|c|c|c|c|c|c|c|c|c|}
\hline $\begin{array}{l}\text { Portafolio } \\
\text { simulado }\end{array}$ & Constante & IPC сотр & MEX_Guber & $\begin{array}{r}\text { MEX } \\
\text { RPORATIVO }\end{array}$ & $\begin{array}{r}\text { Citi-ESBI- } \\
M E X\end{array}$ & Citi-WBI & MSCI-Gross & \multicolumn{2}{|c|}{ UBS-Bcomm Std. error. } \\
\hline IPCcomp & $-0.0002 * * *$ & $0.0688^{*}$ & $-0.3136 * * *$ & $-0.6168 * * *$ & -0.1727 & 0.2219 & $-0.0146^{* * *}$ & $-0.0306 * * *$ & $0.5965 \%$ \\
\hline$I P C$ & -0.0007 & 0.2839 & $0.0026 * * *$ & $-0.1586 * *$ & 0.2856 & -0.1123 & 0.1338 & 0.0231 & $0.0572 \%$ \\
\hline$I P C S$ & -0.0003 & 0.322 & -0.585 & -1.3831 & 0.1068 & $-0.083 * * *$ & $0.0384 * *$ & $-0.0285^{*}$ & $0.1600 \%$ \\
\hline
\end{tabular}

NOTA: El número de asteriscos indican no significancia estadística de los parámetros de la regresora a $1 \%(*), 5 \%(* *)$ y $10 \%(* * *)$ de probabilidad. 
En este punto es de necesidad resaltar que los índices accionarios mexicanos (sustentables o no) tienen un nivel bajo de explicación en el desempeño de las inversiones realizadas en este tipo de portafolio. La razón de ello se encuentra, en parte, en la figura 1, que presenta las probabilidades suavizadas ${ }^{10}$ de estar en periodos de crisis. En el panel superior de la misma se presenta una superficie gris que expone las probabilidades de que los Estados Unidos se encuentren en una recesión, según los cálculos realizados por el National Bureu of Economic Research o NBER (Federal Reserve of Saint Luis, 2016) ${ }^{11}$. En las líneas de la misma se exponen las probabilidades de encontrarse (en el tiempo t) en periodos de crisis $(k=2)$ en cada uno de los tres portafolios de mínima varianza simulados, según lo determinado con el filtro de Hamilton (1989). Lo propio se hace en el panel inferior para los portafolios seleccionados al maximizar el índice de Sharpe.

Como resultado de dicha figura, se puede apreciar primero que, en el caso de los portafolios de mínima varianza, la cartera que empleó el IPCcomp prácticamente solo una vez migró al régimen de crisis; contrario a lo que se observa en el IPCS e IPC en los que su probabilidad de estar en periodos de alta volatilidad es inversa a la probabilidad de estar en recesión en los Estados Unidos. Por otro lado,en los portafolios con el máximo índice de Sharpe, se puede observar que la probabilidad de estar en un periodo de crisis es mayor en el IPCcomp e IPC y su valor es ajeno a los ciclos económicos estadounidenses. Esto debido a que cada tipo de activo financiero tiene sus propios periodos de crisis o fluctuaciones pertenecientes a $k=2$ que pueden no ser debidas a cuestiones netamente económicas; sino propias de la operativa e información corporativa o microeconómica de sus propios mercados.

\footnotetext{
${ }^{10}$ Calculadas al aplicar el filtro de Hamilton en periodicidad mensual

${ }^{11}$ La razón de utilizar estas probabilidades y no las fechas de recesión publicadas por el NBER se fundamentan en los comentarios de Chauvet(2000), quien propone su cálculo como un indicador "adelantado en tiempo real" que no emplea información rezagada o realiza un análisis ex post de los ciclos económicos. Se utilizó la probabilidad de recesión en los EEUU debido a que la misma marcó las probabilidades de los ciclos económicos de México en el periodo estudiado.
} 
Figura 1 Comparativo de las probabilidades de estar en recesión del NBER y las probabilidades suavizadas calculadas al emplear el filtro de Hamilton (1989) en los tres portafolios simulados (IPC, IPCcomp e IPCS) tanto los de mínima varianza como los del máximo índice de Sharpe.
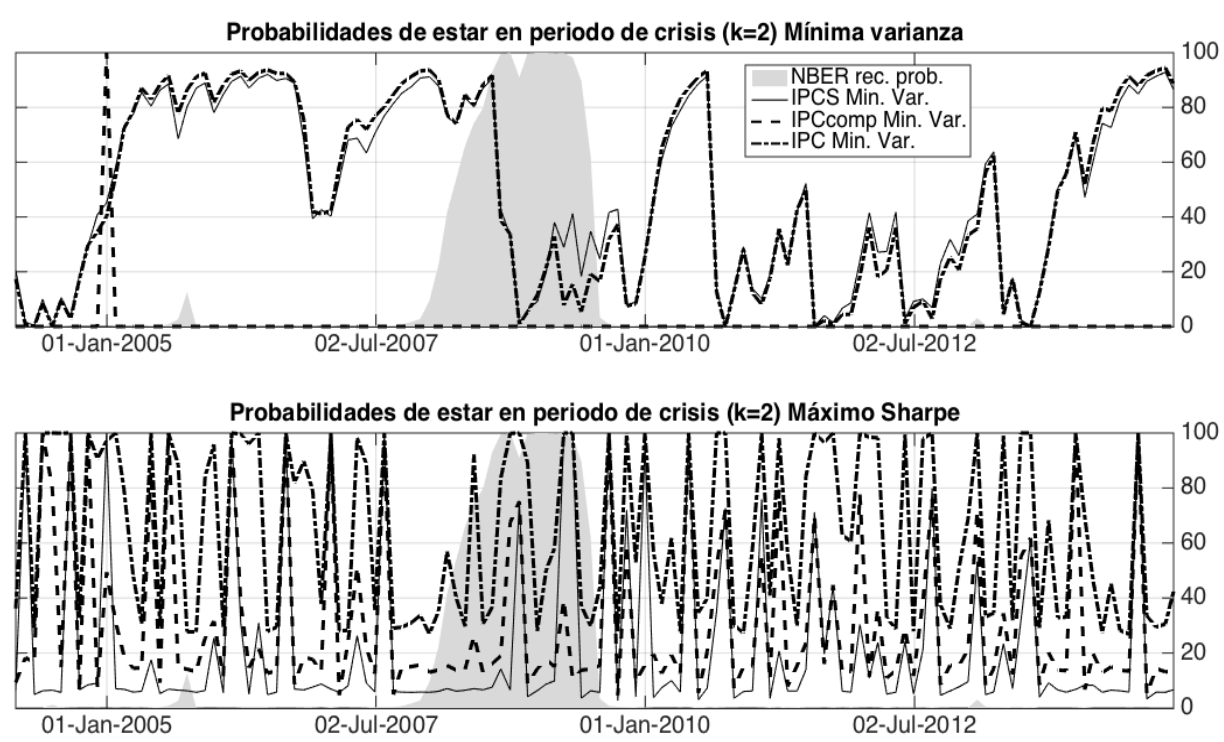

Fuente: Elaboración propia en base a las simulaciones realizadas.

Dado esto, el panel superior apoya la posición tomada de favorecer el empleo de la inversión socialmente responsable en las SIEFOREs debido a que prácticamente la probabilidad de que el portafolio de mínima varianza esté en crisis es sistemáticamente baja.

En el panel inferior de dicha figura se puede apreciar un comportamiento más volátil en el histórico de las probabilidades de estar en crisis para los tres portafolios. Esto es de esperarse ya que el nivel de exposición al riesgo tiende a ser mayor que el tipo de portafolio previamente descrito. Sin embargo, resalta que las probabilidades de estar en crisis también son sistemáticamente más bajas en el caso que emplea el IPCS como factor de renta variable.

Ante estos resultados, se puede apreciar, como sugerencia en términos de política pública y a la luz de estos primeros resultados, que la inversión socialmente responsable aplicada a fondos de pensiones como SIEFOREs, resulta atractiva en términos de rendimiento esperado, de los beneficios sociales y ambientales que se fomentarían, así como en función de los niveles de eficiencia media-varianza que se observaría en el desempeño de las SIEFOREs en periodos convencionales y en los de alta volatilidad. De manera análoga a lo previamente dicho, se puede apreciar que tanto el IPC como el IPC sustentable tienen un notable incremento en su influencia en periodos de alta volatilidad, junto con los bonos gubernamentales mexicanos denominados en dólares. Incluso se puede apreciar la fuerte influencia que la inversión socialmente responsable ejerce en la SIEFORE "tipo" simulada en periodos de crisis. Si esto lo complementamos con el resultado observado en la tabla 6 para los portafolios que maximizan el índice de Sharpe (IPCS en concreto), se llega a la consideración final de que la inversión socialmente responsable no solo lleva a resultados estadísticos similares a la inversión convencional dada con el IPCcomp o la enfocada a empresas de alta capitalización y bursatilidad (IPC); sino que tiene mejores prestaciones de 
eficiencia media-varianza en periodos de crisis. Ante esto, es de necesidad resumir los resultados estudiados en el siguiente corolario:

1. Los portafolios de mínima varianza tienen desempeños similares debido a que los índices accionarios mexicanos tienen baja influencia en su desempeño. Dado esto y la similitud estadística, puede decirse que resulta de interés enfocar los esfuerzos de inversión en las carteras que maximizan el índice de Sharpe.

2. Dado lo anterior y los resultados de la tabla 6, se aprecia que, si se emplea la inversión socialmente responsable en acciones mexicanas, se tienen niveles altos y adecuados de rendimiento esperado en periodos de convencionales de volatilidad, pero, en tiempos de mayor turbulencia, la inversión socialmente responsable en el régimen e inversión de una SIEFORE resulta más atractiva en términos de rendimiento esperado que la inversión convencional.

3. Si se extiende esta consideración a los niveles de exposición al riesgo y, como consecuencia, a los niveles de eficiencia media-varianza medidos con el índice de Sharpe, se puede apreciar que resulta más adecuado invertir en acciones socialmente responsables. Esto no solo por su pertinencia por el tipo de activos objeto de inversión; sino por su relación de rendimiento esperado y exposición al riesgo en tiempos de crisis o alta volatilidad.

4. Se sugiere, ante los resultados de la tabla 6, la posibilidad de tener una inversión dinámica entre IPC e IPCS. Esto porque el IPCS tiene mejor eficiencia mediavarianza en periodos de alta volatilidad y el IPC en los de baja. Esto sugiere que la responsabilidad social podría no estar del todo descontada por los inversionistas en el mercado mexicano, resultado que podría incentivarmás estudios en la materia.

\section{Conclusiones.}

La responsabilidad social empresarial es una materia de amplio interés en las ciencias económico-administrativas por la serie de recomendaciones que se hacen a la empresa en términos de su relación no solo con los inversionistas; sino también con las partes interesadas (stake holders) como son los propios trabajadores, proveedores, sociedad en general y medio ambiente. Ante esto y como resultado de las prácticas de inversión socialmente responsable (o inversión sustentable), surge la necesidad de enfocar estudios que vean la pertinencia de realizar este nuevo "estilo de inversión".

Para estudiar dicho beneficio, se recalculó en el presente trabajo el IPC sustentable a enero de 2004. Lo anterior se realizó debido a que el índice original cotiza desde noviembre de 2008. Para lograr este cálculo,se utilizó el desempeño histórico de las acciones miembro al día 28 de noviembre de 2008 y se empleó el método de capitalización como método de ponderación (primera aportación del trabajo). Como resultado de esto, se empleó una política de inversión general para una SIEFORE tipo, misma que pudiese aproximar el desempeño de "todas" las SIEFOREs (independientemente del tipo) y que se basara en la política de inversión autorizada por la CONSAR (2016). Lo único que se hizo en las simulaciones fue sustituir, en tres escenarios diferentes, el índice de acciones mexicanas a emplear. Como candidatos se utilizó el IPCcomp de 60 acciones, el benchmark más empleado por los profesionales dado con el IPC (35 acciones) y el IPC sustentable (de 5 a 35 acciones según el resultado del rebalanceo). Para simular la política de inversión en estos tres escenarios, se calculó el portafolio de mínima varianza y el que maximiza el índice de Sharpe.

Los resultados observaron que los portafolios de mínima varianza no presentan un desempeño significativamente diferente, dado que la inversión en acciones mexicanas es 
Nueva Época REMEF (TheMexicanJournal of Economics and Finance)

muy baja y no tiene una influencia significativa (más aún en periodos de alta volatilidad). Sin embargo, para el caso de los portafolios que maximizan el índice de Sharpe, se observó que el invertir en acciones socialmente responsables no lleva a pérdidas significativas de eficiencia media varianza y que incluso, en periodos de alta volatilidad en los mercados o crisis, su rendimiento esperado y eficiencia media-varianza tienden a ser superior, en comparación a lo que sucedería si emplea la inversión "convencional" representada por el IPC o el IPCcomp.

Ante estos resultados, se puede apreciar, como sugerencia en términos de política pública y a la luz de estos primeros resultados, que la inversión socialmente responsable realizada por fondos de pensiones (como son las SIEFOREs) resulta atractiva en términos de rendimiento esperado y eficiencia media-varianza, así como en términos de los beneficios sociales que conlleva el destinar capital a los valores de empresas que tienen la cualidad de ser socialmente responsables. Esto deja la puerta abierta a una línea de estudio que puede desarrollarse en beneficio de las y los ahorradores de un sistema de pensiones, así como para dar apoyo a la cultura de una mayor responsabilidad social en las empresas. Lo último por medio del incentivo de destinar más capital a aquellas empresas o emisores que "mejor se portan" y son, en términos de la perspectiva del presente trabajo, socialmente responsables.

\section{Referencias}

Areal, N., Cortez, M. C. and Silva, F. (2013). The conditional performance of US mutual funds over different market regimes: do different types of ethical screens matter? Financial markets and portfolio management, 27(4), pp. 397-429. http://doi.org/10.1007/s11408-013-0218-5

Bauer, R., Koedijk, K. and Otten, R. (2005). International evidence on ethical mutual fund performance and investment style. Journal of Banking \& Finance, 29(7), 1751-1767.

Berry, T. C. and Junkus, J. C. (2013). Socially Responsible Investing: An Investor Perspective. Journal of Business Ethics, 112(4), pp. 707-720. http://doi.org/10.1007/s10551-012-1567-0

Bodie, Z., Kane, A., and Marcus, A. (2014). Investments global edition (10th ed.). New York, USA: Mc Graw-Hill.

Boutin-Dufresne, F., and Savaria, P. (2004). Corporate Social Responsibility and Financial Risk. The Journal of Portfolio Management, 13(1), pp. 57-66.

Carhart, M. M. (1997). On Persistence in Mutual Fund Performance. The Journal of Finance, LII(1), 57-82.

Chauvet, M. (2000). An econometric characterization of business cycle dynamics with factor structure and regime switching. International Economic Review, 10(2), pp. 127142.

CONSAR. (2012). Disposiciones de carácter general que establecen el régimen de inversión al que deberán sujetarse las sociedades de inversión especializadas de fondos para el retiro. Recuperado el3 de febrero de 2013, de: http://www.consar.gob.mx/normatividad/normatividad-normatividad_consarcirculares.shtml

CONSAR. (2016). Disposiciones de carácter general que establecen el régimen de inversión al que deberán sujetarse las sociedades de inversión especializadas de fondos para el retiro. Recuperado el 3 de febrero de 2015, de: http://www.consar.gob.mx/normatividad/pdf/normatividad_emitida/circulares/Disposi 
ciones_de_caracter_general_regimen_de_inversi?n_20160104.pdf

De la Torre, O., Galeana, E. y Aguilasocho, D. (2015). The use of the Sustainable Investment against the broad market one. A first test in the Mexican stock market. Investigaciones Europeas de Dirección y Economía de Empresa, 22(13), pp. 117123.http://dx.doi.org/10.1016/j.iedee.2015.08.002

De la Torre, O. y Martínez Ma Isabel. (2015). Revisión de la inversión sustentable en la bolsa mexicana durante periodos de crisis. Revista Mexicana de Economía y Finanzas, 10(2), pp. 115-130. Recuperado de: http://www.remef.org.mx/c/images/uploads/documentos/134/10_2_2_maria1.pdf

Eccles, N. S. and Viviers, S. (2011). The Origins and Meanings of Names Describing Investment Practices that Integrate a Consideration of ESG Issues in the Academic Literature. Journal of Business Ethics, 104(3), pp. 389-402. http://doi.org/10.1007/s10551-011-0917-7

Fama, E. F. and French, K. R. (1992). Common risk factors in the returns on stocks and bonds. Journal of Financial Economics, 33(1), pp. 3-56.

Federal Reserve of Saint Luis. (2016). Smoothed U.S. Recession Probabilities (RECPROUSM156N). Recuperado el 13 de September de 2016, de: https://fred.stlouisfed.org/series/RECPROUSM156N

Hamilton, J. D. (1989). A New Approach to the Economic Analysis of Nonstationary Time Series and the Business Cycle. Econometrica, 57(2), pp. 357-384.

Hamilton, J. D. (1994). Time series analysis. Princeton: Princeton university press.

Hongbo, H., Mitchell, O. and Piggott, J. (2006). Socially responsible investment in Japanese Pensions. Pacific-Basin Finance Journal, 14, pp. 427-438. http://doi.org/10.1016/j.pacfin.2006.03.002

Huberman, G. and Kandel, S. (1987). Mean-Variance Spanning. The Journal of Finance, 42(4), pp. 873-888. http://doi.org/10.2307/2328296

Moskowitz, M. (1972). Choosing socially responsible stocks. Business and Society Review, 1(1), 71-75. article.

Renneboog, L., Ter Horts, J. and Zhang, C. (2011). Is ethical money financially smart? Nonfinancial attributes and money flows of socially responsible investment funds. Journal of Financial Intermediation, 20(2011), pp. 562-588.

Schröder, M. (2004). The performance of socially responsible investments: investment funds and indices. Financial Markets and Portfolio Management, 18(2), pp. 122-142.

Schröder, M. (2007). Is there a Difference? The Performance Characteristics of SRI Equity Indices. Journal of Business Finance and Accounting, 34(1-2), pp. 331-348.

Sethi, S. P. (2005). Investing in socially responsible companies is a must for public pension funds - Because there is no better alternative. Journal of Business Ethics, 56, 99-129. http://doi.org/10.1007/s10551-004-5455-0

Statman, M. (2000). Socially Responsible Mutual Funds. Financial Analysts Journal, 56(3), pp. 30-39.

United Nations. (2000). Resolution 2 session 55 United nations Millennium Declaration.

United Nations. Resolution 60/1. 2005 world summit outcome (2005). New York: United Nations. Recuperado

de: http://data.unaids.org/Topics/UniversalAccess/worldsummitoutcome_resolution_24oct 2005_en.pdf

Valencia, H. (2015). Decomposition of the stocks returns in the sustainable index of the Mexican Stock Exchange Decomposition of the stocks returns in the sustainable index 
Nueva Época REMEF (TheMexicanJournal of Economics and Finance)

of the Mexican Stock Exchange. Revista Mexicana de Economía y Finanzas, 10(1), pp. 85-99. 